\title{
STEM-EDX AND FIB-SEM TOMOGRAPHY OF ALLVAC 718PLUS SUPERALLOY
}

\begin{abstract}
Allvac 718Plus (718Plus) is a high strength, corrosion resistant nickel- based superalloy used for application in power generation, aeronautics and aerospace industry. The 718Plus microstructure consists of a $\gamma$ matrix with $\gamma^{\prime}-\mathrm{Ni}_{3}(\mathrm{Al}, \mathrm{Ti})$ and some $\delta-\mathrm{Ni}_{3} \mathrm{Nb}$ phases as well as lamellar particles $\left(\eta-\mathrm{Ni}_{3} \mathrm{Ti}, \eta^{*}-\mathrm{Ni}_{6} \mathrm{AlNb}\right.$ or $\left.\mathrm{Ni}_{6}(\mathrm{Al}, \mathrm{Ti}) \mathrm{Nb}\right)$ precipitated at the grain boundaries. The primary strengthening mechanism for this alloy is a precipitation hardening, therefore size and distribution of precipitates are critical for the performance of the alloy.

The aim of this study was to characterize precipitates in the 718Plus superalloy using Scanning Transmission Electron Microscope combined with Energy Dispersive X-ray Spectroscopy (STEM-EDX) and Focused Ion Beam Scanning Electron Microscope (FIB-SEM). The STEM-EDX and FIB-SEM tomography techniques were used for 3D imaging and metrology of the precipitates. Transmission electron microscopy and EDX spectroscopy were used to reveal details of the 718Plus microstructure and allow determine chemical composition of the phases. The study showed that electron tomography techniques permit to obtain complementary information about microstructural features (precipitates size, shape and their 3D distribution) in the reconstructed volume with comparison to conventional particle analysis methods, e.g. quantitative TEM and SEM metallography.
\end{abstract}

Keywords: TEM, SEM, STEM-EDX tomography, FIB-SEM tomography, Allvac 718Plus

\section{Introduction}

Allvac 718Plus (718Plus) is a high strength, corrosion resistant nickel-based superalloy designed to replace Inconel 718 (IN718) in static and rotating application in power generation, aeronautics and aerospace industry [1-3]. This alloy is mainly used for turbine discs and vanes in aero-engines and industrial gas turbines. The 718Plus superalloy shows improved temperature performance, compared to the Inconel 718, while retaining the excellent processing characteristics of that alloy. The main differences in chemistry between IN718 and 718Plus superalloys ware addition of $\mathrm{Co}$, lower Fe content as well as lower Ti to Al ratio. Its typical chemical composition is the following: $\mathrm{Ni}-18 \mathrm{Cr}-10 \mathrm{Fe}-9 \mathrm{Co}-5.1(\mathrm{Nb}+\mathrm{Ta})-1 \mathrm{~W}-2.7 \mathrm{Mo}-0.7 \mathrm{Ti}-$ $1.5 \mathrm{Al}-0.03 \mathrm{C}$ (wt.\%). The 718Plus microstructure consists of a $\gamma$ matrix (Ni-based solid solution) with ordered face centred cubic $\gamma^{\prime}-\mathrm{Ni} 3(\mathrm{Al}, \mathrm{Ti})$ type phase, some orthorhombic $\delta-\mathrm{Ni}_{3} \mathrm{Nb}$ and hexagonal $\eta-\mathrm{Ni}_{3} \mathrm{Ti}, \eta^{*}-\mathrm{Ni}_{6} \mathrm{AlNb}$ or $\mathrm{Ni}_{6}(\mathrm{Al}, \mathrm{Ti}) \mathrm{Nb}$ particles precipitated mainly at the grain boundaries [4-7]. This alloy is strengthened by a high volume fraction of the ordered $\gamma^{\prime}$ phase, precipitated coherently in $\gamma$ matrix. The change of main strengthening phases from $\gamma^{\prime \prime}+\gamma^{\prime}$ phases for IN718 to the $\gamma^{\prime}$ phase for 718 Plus provides greater stability at high temperatures. The manufacturers of 718Plus alloy claim an increased service temperature of $718 \mathrm{Plus}$ in comparison to IN718 alloy of $55{ }^{\circ} \mathrm{C}$ and a maximum service temperature of $704{ }^{\circ} \mathrm{C}$ [8]. According to the Allvac data sheet [8], the hardness of the 718Plus is nominally $20-30 \mathrm{HRC}$, but in the fully aged condition reaches $38-45 \mathrm{HRC}$, it means an increase of about $50 \%$. The linear coefficient of thermal expansion of the 718 Plus is below $2 \times 10^{-5}{ }^{\circ} \mathrm{C}^{-1}$ at $900{ }^{\circ} \mathrm{C}$, its creep resistance at $880^{\circ} \mathrm{C}$ is three times higher, compared to alloy IN718 [9].

The development of innovative structural materials for energy systems and aeronautics requires application of modern research methods to qualitative and quantitative characterization of the microstructure on the level from a micro- to nanoscale.

Modern imaging techniques available by SEM, TEM allow to apply tomographic techniques to characterize the materials microstructure in the three-dimensions [10, 11]. Application of these imaging methods, relatively new in material science, enables to estimate the influence of the microstructural parameters on mechanical and corrosion properties of modern structural materials with high accuracy that cannot be obtained by "conventional methods".

Scanning and Transmission Electron Microscopy methods provide the qualitative and quantitative information about the phase composition and their morphology in engineering materials. These methods are based on metrology of the 2D projections of the $3 \mathrm{D}$ objects. Application of tomography techniques, such as TEM and FIB-SEM tomography provides new opportunities. These techniques allow to reconstruct the microstructure of investigated materials in three dimensions (3D). TEM-Bright Field (TEM-BF) images which are 2D projections of the sample volume give valuable information about the microstructure and chemical composition of materials.

* AGH UNIVERSITY OF SCIENCE AND TECHNOLOGY, INTERNATIONAL CENTRE OF ELECTRON MICROSCOPY FOR MATERIALS SCIENCE, FACULTY OF METALS ENGINEERING AND COMPUTER INDUSTRIAL SCIENCE, AL. MICKIEWICZA 30, 30-059 KRAKOW, POLAND

\# corresponding author: kruczek@agh.edu.pl 
The electron tomography was developed to reconstruct objects in three dimensions (3D) from a tilt series of TEM or a series of cross-sections in FIB-SEM images. The TEM and FIBSEM tomography techniques have been successfully adopted by researchers to materials science $[12,13]$. The resolution (determined as a voxel size) in $3 \mathrm{D}$ reconstructions, however, depends on applied tomographic technique and varies from tenths parts to few tens of nanometers.

STEM electron tomography procedure can be divided into 4 steps. At first, a series of $2 \mathrm{D}$ projections (images) of the specimen are semi automatically recorded using software dedicated for this purpose (Explore 3D software, FEI). Specimen is systematically tilted in the microscope to different angles in the range of $\pm 75^{\circ}$ using a special tomographic holder. During acquisition of an image series a shift adjustment between consecutive images is performed. In a second step, these individual images are aligned using Inspect 3D (FEI) software (off-line processing) to a common tilt axis origin. In the next step, 3D volume is reconstructed using for example Back Projected method (BP) to create a 3D representation of the sample in the Fourier space. The Direct Fourier (DF) method and the Filtered Back Projection method (WBP) are two of the most used heuristics for tomographic reconstruction. They are fast methods of reconstruction, but in many cases the results contain several artifacts. A better reconstruction results are achieved using iterative methods such as Algebraic Reconstruction Techniques (ART) and Simultaneous Iterative Reconstructive Technique (SIRT). The last step is connected with $3 \mathrm{D}$ visualization and metrology of reconstructed volume of the sample. A requirement for the calculation of $3 \mathrm{D}$ reconstructions based on electron micrographs in TEM tomography is that the registered image contrast must be a projection of some physical characteristic of the sample, e.g. its mass/electron density (Z-contrast). This implies that conventional electron tomography using TEM Bright-Field (TEM-BF) or Dark-Field (TEM-DF) imaging, dominated by diffraction and/or scattering contrast cannot be used. This problem can be overcome by acquisition of projection images by STEM, high angular annular dark-field (HAADF) imaging mode utilizing Z-contrast. Although these methods are very powerful, an exposure (scanning) time of 10 - 30 sec. per image is required when using STEM - HAADF mode which results in scanning noise. Several different modes of image acquisition can be utilized in TEM tomography to give insight into different characteristics of the sample. One of the newer imaging methods is the STEM X-ray spectroscopic imaging (STEM-EDX), which enables the mapping of local concentrations of selected chemical elements.

Recently FIB in conjunction with SEM has become an important tool in materials science for studying materials at the micro- and nanometer scale. FIB-SEM tomography is based on a serial sectioning and imaging technique employing a FIB-SEM dual beam workstation. Dual-beam SEM enables the acquisition of serial images with small (few nanometers) and reproducible spacing between the single imaging planes. In many FIB-SEM facilities no mechanical stage tilting is necessary between the FIB milling and the electron beam imaging steps. Application of computer algorithms for experimental raw data stack processing (e.g. ImageJ 1.45b) and graphics packages (e.g. Amira 5.4.1) for 3D visualization allows to obtain both qualitative and quantitative information about reconstructed volume. It is possible to study several features at spatial resolutions of tens of nanometers and volumes up to tens of cube microns.

In this study both tomography techniques described above, i.e. electron STEM-EDX and FIB-SEM tomography were applied for 3D imaging and metrology of the strengthening particles in 718Plus superalloy. SEM and TEM as well as STEM-EDX spectroscopy were used to reveal details of superalloy microstructure and chemical composition of the phases.

\section{Experimental procedure}

The investigated 718Plus superalloy was as follows: Ni$18 \mathrm{Cr}-10 \mathrm{Fe}-9 \mathrm{Co}-5.1(\mathrm{Nb}+\mathrm{Ta})-1 \mathrm{~W}-2.7 \mathrm{Mo}-0.7 \mathrm{Ti}-1.5 \mathrm{Al}-0.03 \mathrm{C}$ wt.\%. Microstructural investigation and phase chemical composition of the 718Plus were performed using conventional TEM (BF, DF) imaging and electron diffraction analyses as well as STEM-EDX techniques. The TEM study was performed on the lamella prepared by FIB (NEON CrossBeam 40EsB) using Tecnai G2 20 TWIN and a probe Cs corrected Titan Cubed G2 60-300 with ChemiSTEM ${ }^{\mathrm{TM}}$ system with Super-X detector [14]. The EDX detection system allowed to achieve high level of X-ray signal over a large tilt angle of sample and collect series of 2D elemental maps in the angular range from $-60^{\circ}$ to $+60^{\circ}$ with step of $4^{\circ}$. Pre-processing and alignment of image tilt series were performed using TomoJ 2.12 software. Tomographic reconstruction of a tilt series of 31 images (elemental maps) was performed using SIRT method with number of iteration equal 12, which allowed visualizing the three-dimensional distribution of selected elements (Al, $\mathrm{Cr}$ ) in the analysed volume.

The other tomography technique used for 3D imaging of precipitates in the 718Plus microstructure was FIB-SEM tomography. The NEON CrossBeam 40EsB with Ga-ion beam at parameters: $30 \mathrm{kV}, 200 \mathrm{pA}$ and aperture of $30 \mu \mathrm{m}$ was used to perform a precise in-situ milling. Fig. 1 shows the preparation of the ROI (Region of Interest) in examined sample for tomographic image acquisition. The Pt protective layer was deposited on the sample surface (top surface of ROI) in order to prevent the occurrence of the curtaining effect, i.e., the irregular removal of material. Consequently, the acquired stack of images (image size 1024 x 768 pixels, 599 images in 8-bit grayscale, stack size $462 \mathrm{MB}$ ) was transformed directly into a 3D data volume with a voxel size of $12 \times 12 \times 12 \mathrm{~nm}$. For microstructure imaging in SEM the Energy selective Backscattered (EsB) electron detector was used. The EsB detector is suitable for clear compositional contrast. Sequential removal of layers as thin as a $12 \mathrm{~nm}$ allowed exploring a total volume of $10.7 \times 7.2 \times 7.7 \mu \mathrm{m}\left(\mathrm{V}=593.2 \mu \mathrm{m}^{3}\right)$. The shift of the electron beam in lateral direction due to slice cutting during recording of the tomogram was calculated and subsequently corrected with the ImageJA $1.45 \mathrm{~b}$ software. From the series of SEM micrographs, the $3 \mathrm{D}$ reconstruction of the investigated alloy volume was generated by Avizo Fire 6.3 software. This program applies the "labelling by reconstruction" technique based on the "neighbourhood-algorithm". This algorithm comprises neighbouring voxels with the same grayscale to local 
objects. Non continuous ranges were identified and marked by different colours. The $3 \mathrm{D}$ visualization of reconstructed space for FIB-SEM tomography was performed using ImageJA $1.45 \mathrm{~b}$ and Avizo Fire 6.3 software.

\section{Results and discussion}

The Allvac 718Plus superalloy has a different microstructure in comparison to the conventional IN718 alloy. The strengthening phase of 718Plus alloy are $\gamma^{\prime}$ particles (rather than $\gamma^{\prime}$ and $\gamma^{\prime \prime}$ ), while $\gamma^{\prime \prime}$ is the main strengthening phase in IN718 superalloy. According to [7], the $\gamma^{\prime}$ phase shows a lower tendency to coarsening and, therefore, is more stable than the $\gamma^{\prime \prime}$ phase, what contributes to a $55^{\circ} \mathrm{C}$ temperature advantage with a maximum service temperature of $704^{\circ} \mathrm{C}$ in comparison to the IN718 [8]. In Allvac 718Plus, the non-uniform precipitation of $\eta$-like platelets was observed along the grain boundaries and as lamellar precipitates inside the $\gamma$ grains. SEM-EDX and STEM-EDX techniques were used to obtain qualitative information (maps) about chemical composition of the precipitates. The results showed that $\mathrm{Ni}, \mathrm{Al}, \mathrm{Ti}$ and $\mathrm{Nb}$ partition to the $\eta$ phase. The hexagonal crystal structure of the lamellar phase suggested the $\eta-\mathrm{Ni}_{3} \mathrm{Ti} \mathrm{D}_{24}$ phase but its chemistry was close to the $\mathrm{Ni}_{6}(\mathrm{AlTi}) \mathrm{Nb}$ [4]. Pickering et al [4] reported discontinuous precipitation of the $\mathrm{Ni}_{6}(\mathrm{AlTi}) \mathrm{Nb}$ phase. In our study, a morphology of the $\eta$-like phases support this precipitation mechanism.

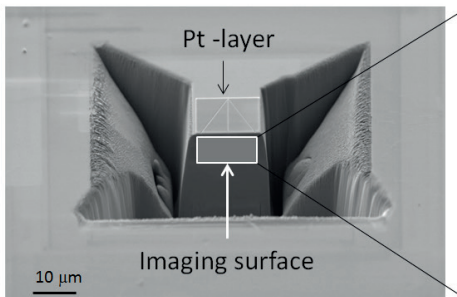

a)

Fig. 1. Geometry of the sample prepared for serial FIB sectioning; (a) preparation of ROI for tomographic imaging, SEM-SE, (b) $\eta$ phase precipitates at the $\gamma$ grain boundary (intergranular precipitates), SEMBSE image

TEM investigations of the 718Plus revealed that its microstructure consists of the $\gamma$ matrix strengthened by ordered face centred cubic $\gamma^{\prime}$ phase precipitates and the $\eta$ phase particles of hexagonal crystal structure. Fig.2. shows colonies of lamellar $\eta$ phase precipitated at the $\gamma$ grain boundaries and inside the $\gamma$ grains as well as the $\gamma^{\prime}$ spherical precipitates.

Based on performed structural studies using TEM and SEM, it can be concluded that intergranular precipitate at the $\gamma$ grain boundary appeared to have an orientation relationship with the parent $\gamma$ grain. This indicates that $\eta$ phase nucleation has occurred at the $\gamma$ grain boundary and the $\eta$ precipitate can grow forwards by a discontinuous mechanism of precipitation into the unrelated grain as lamellar-like precipitates or backwards into the grain in which it nucleated. Although most of the precipitates had a similar morphology, some variations were observed in the lamellar spacing, thickness and length of the individual precipitates. Determination of the exact shape and dimensions of the $\eta$ phase precipitates based on 2D projection is rather difficult. Strengthening precipitates of the $\gamma^{\prime}$ phase in the $\gamma$ matrix are shown in Fig. 3a by a weak contrast around particles, which is caused by lattice strain at the matrix/ precipitate interfaces. Fig. 3 shows the precipitates as seen by different imaging modes (TEM-BF, STEM-HAADF and SEM-BSE); homogenously distributed, nearly spherical $\gamma^{\prime}$ and lamellar $\eta$ phase precipitates are shown in Fig. $2 d$, Fig. $3 b$ and c. STEM-EDX elemental maps of $\mathrm{Ni} \mathrm{K} \alpha, \mathrm{Nb} \mathrm{K} \alpha, \mathrm{Al} \mathrm{K} \alpha$ and $\mathrm{Ti}$ $\mathrm{K} \alpha$ give the possibility to obtain $\gamma^{\prime}$ and $\eta$ phase imaging during acquisition of tilt series of images in angular range $\pm 60^{\circ}$ with step of $4^{\circ}$
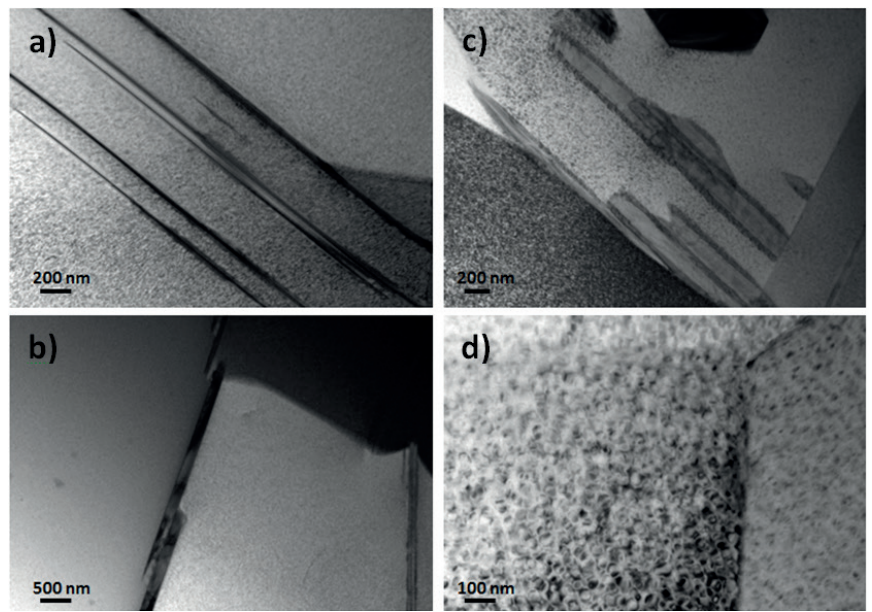

Fig. 2. Microstructure of 718Plus superalloy; a) precipitates of $\eta$ phase inside the $\gamma$-phase grain, b) precipitates of $\eta$ phase at the $\gamma$-phase grain boundary (intergranular precipitates), c) precipitates of $\eta$ phase inside the $\gamma$ phase and at the $\gamma$ grain boundary d) strengthening $\gamma^{\prime}$ particles in $\gamma$ matrix. TEM-BF

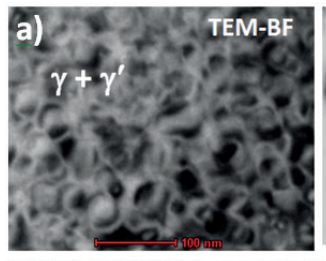

b)

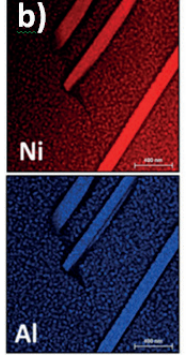

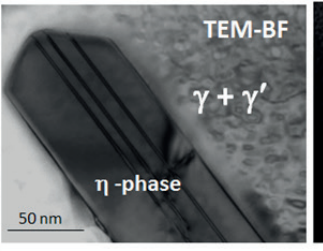

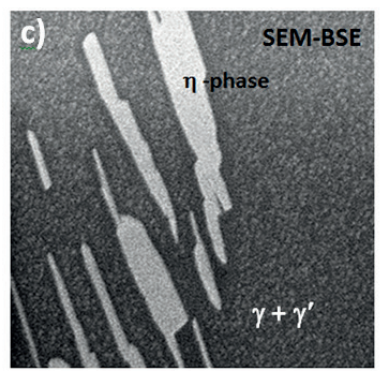

Fig. 3. Microstructure of 718Plus superalloy; a) TEM-BF and STEMHAADF imaging of the 718Plus shows spherical $\gamma^{\prime}$ and platelet $\eta$ phase precipitates. (b) STEM-EDX elemental maps from the area of lamellar precipitates of the $\eta$ phase. c) SEM-BSE image of the 718 Plus microstructure showing the different shape of $\eta$ phase precipitates in the matrix

A series of elemental maps obtained using STEM-EDX of the $\eta$ phase and its surrounding regions are shown in Fig. 4. A tilt series of $\mathrm{Al}$ and $\mathrm{Cr}$ elemental maps were used for STEM- 
EDX tomographic reconstruction and visualization of sample volume from the area selected by the rectangle in Fig.4. Due to the fact that $\mathrm{Al}$ atoms are present in both $\gamma^{\prime}$ and $\eta$ phases ( $\mathrm{Cr}$ is present only in the $\gamma$ phase) application of tomographic reconstruction to registered tilt series of $\mathrm{Al}$ maps enables a qualitative and quantitative analysis of spatial distribution of $\gamma^{\prime}$ particles.
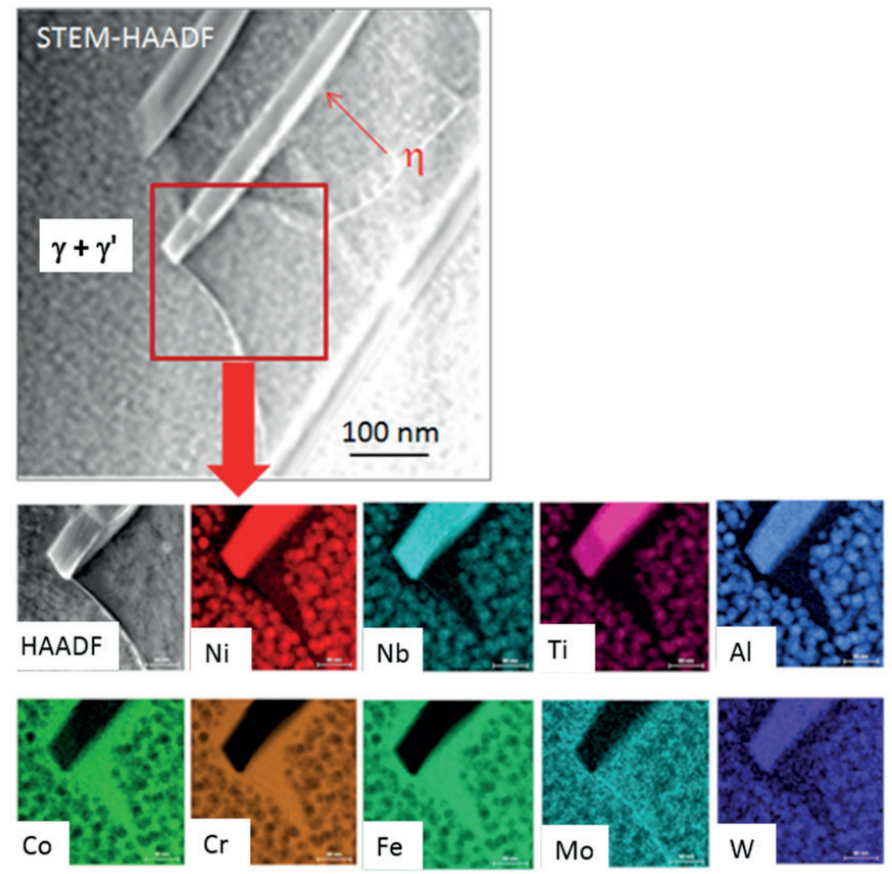

Fig. 4. An example of STEM-EDX elemental maps of selected microstructural elements recorded at $0^{\circ}$ sample tilt from the area marked in a STEM-HAADF image
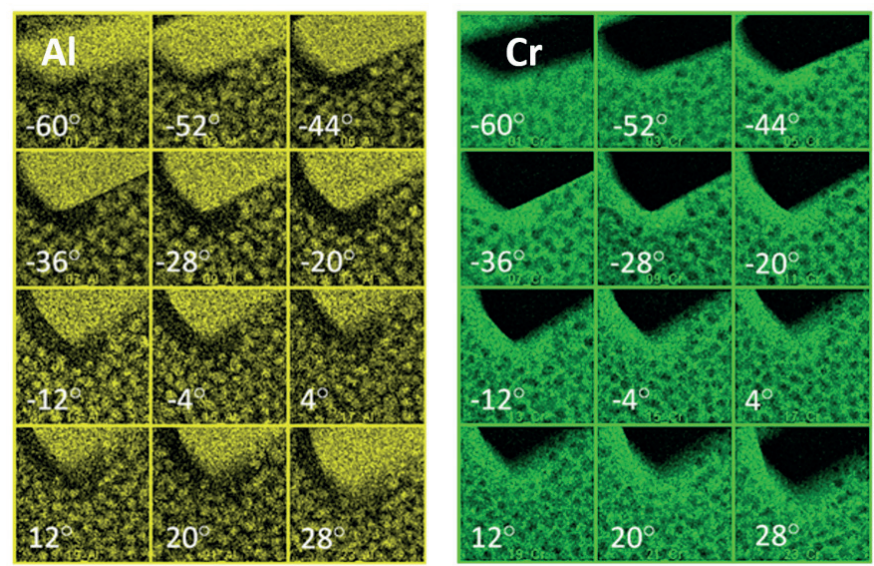

Fig. 5. STEM-EDX elemental maps of $\mathrm{Al}$ and $\mathrm{Cr}$ at different tilt angle selected from the registered tilt series

STEM-EDX elemental $\mathrm{Al}$ and $\mathrm{Cr}$ maps at different tilt angle, selected from the registered tilt series, are shown in Fig.5. The results of 3D visualization of the shape and distribution of $\gamma^{\prime}$ particles in the 718Plus superalloy as well as $\eta$ phase particles at different angle of view using STEM-EDX tomography technique are shown in Fig. 6. The volume fraction $\left(\mathrm{V}_{\mathrm{v}}\right)$ of $\gamma^{\prime}$ phase and the mean equivalent particle diameter $\left(D_{\text {eq }}\right)$ in the reconstructed volume were as follows: $V_{v}=12.5 \%$ and $\mathrm{D}_{\mathrm{eq}}=26.0 \pm 8.3 \mathrm{~nm}$. The distribution of the equivalent diameter $D_{\mathrm{eq}}$ of the $\gamma^{\prime}$ particles in the reconstructed volume of the sample is presented on Fig.7. For the reconstruction STEM-EDX tomography technique was used.

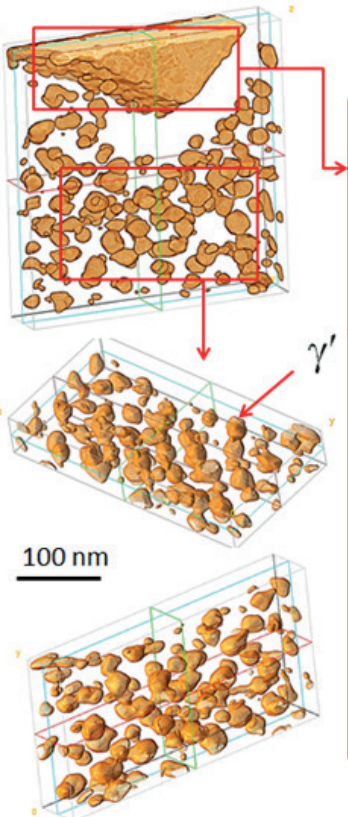

a)

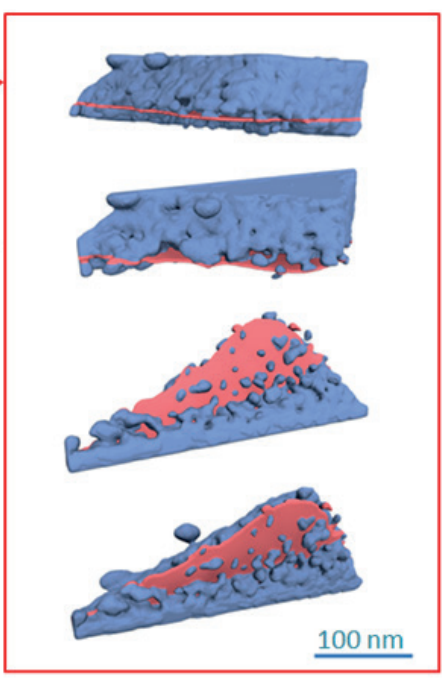

b)
Fig. 6. Visualization of $\gamma^{\prime}$ particles in the 718Plus superalloy. Volume reconstruction was performed using STEM-EDX tomography technique; a) 3D visualization of $\gamma^{\prime}$ and b) $\eta$ phase particle at different angle of view

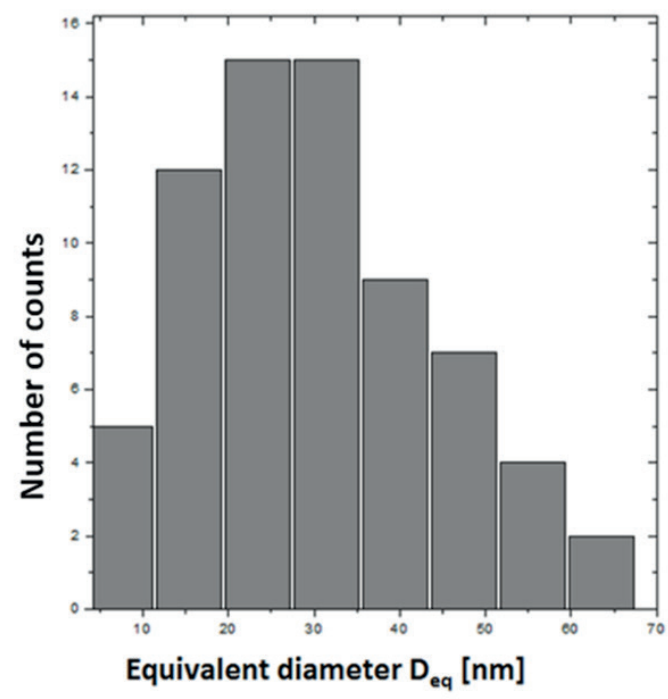

Fig. 7. The distribution of the equivalent diameter Deq of the $\gamma^{\prime}$ precipitates reconstructed using STEM-EDX tomography technique

SEM-EDS and STEM-EDS analyses showed that the $\gamma$ matrix contains $\mathrm{Ni}, \mathrm{Fe}, \mathrm{Co}, \mathrm{Cr}$ and $\mathrm{W}$, while $\mathrm{Ni}, \mathrm{Al}, \mathrm{Ti}$ and $\mathrm{Nb}$ partition to the $\gamma^{\prime}$ and $\eta$ phases (Fig.4).

Microstructure of the 718Plus superalloy using SEMSE and SEM-BSE show $\gamma^{\prime}$ precipitates (SE images) and precipitates of the $\eta$ phase inside the $\gamma$ grain (BSE images), 
Fig.8. The use of digital filtering (band-pass filtering using ImageJ software) to the registered images (SE images) allowed the quantitative evaluation of $\gamma^{\prime}$ phase precipitates. The volume fraction of $\gamma^{\prime}$ phase and the mean equivalent particle diameter in the reconstructed volume determined on 2D images were as follows: $\mathrm{V}_{\mathrm{v}}=13.2 \%$ and $\mathrm{D}_{\mathrm{eq}}=27.5 \pm 8.3 \mathrm{~nm}$. The distribution of the equivalent diameter $\mathrm{D}_{\text {eq }}$ of the $\gamma^{\prime}$ particles observed in SEM-SE images is presented on Fig.9.
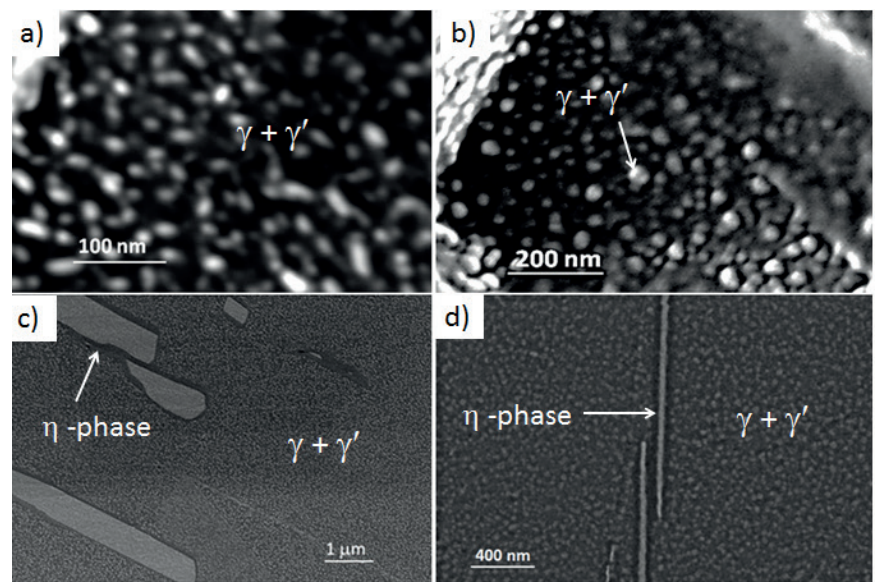

Fig. 8. Microstructure of the 718Plus superalloy a,b) precipitates of $\gamma^{\prime}$ inside the $\gamma$ grain, SEM-SE c,d) precipitates of $\eta$ phase inside the $\gamma$ grain SEM-BSE

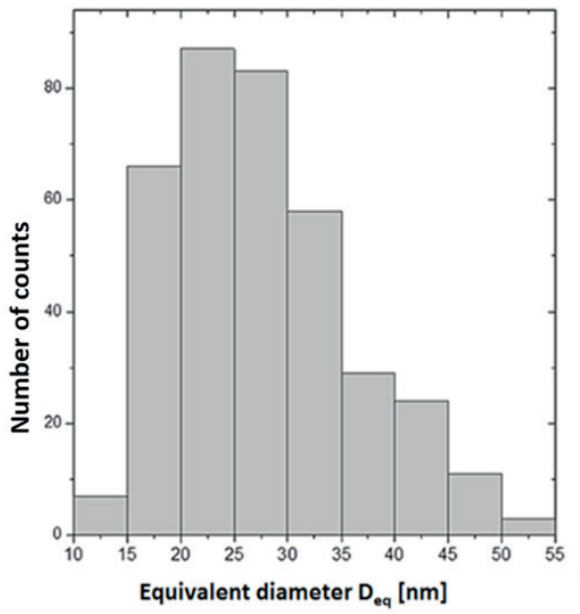

Fig. 9. The distribution of the equivalent diameter Deq of the $\gamma^{\prime}$ precipitates derived from 2D data (SEM-SE images)

Meso-scale FIB-SEM tomography technique was used for detailed characterization of spatial distribution and metrology of the $\eta$ phases and selected carbides in the 718Plus superalloys with resolution of $12 \mathrm{~nm}$. SEM backscattered electrons (BSE) image of the 718Plus superalloy presents the different shape of the $\eta$ phase precipitated in the $\gamma$ matrix. SEM-BSE imaging contrast level in 8-bit grayscale between the $\eta$ phase and $(\gamma$ $\left.+\gamma^{\prime}\right)$ phases (EsB images) allows to perform a tomographic reconstruction of microstructural elements using the FIB-SEM tomography technique. The choice of place for tomographic reconstruction depends on the contrast between elements of the microstructure during SEM-BSE observation. The differences in contrast between grains with different crystallographic orientation result from electron channeling effect. This effect combine imaging and orientation information available in the SEM during imaging at low acceleration voltages in the BSE detector. The channels for electrons created between the atomic centers provide a path for the beam to penetrate deeper into the crystal before elastic scattering. As a result, the backscattering coefficient (the grey level of the image) is reduced when the electron beam is parallel to the crystal channels. In this case, SEM-BSE images are becoming darker. For slightly different beam-crystal orientations, the electron beam scatters more closely to the sample surface, which increases the backscattering coefficient. These areas on BSE images are becoming brighter. The apparent differences in image contrast between $\eta$ particles and different crystallographic oriented grains caused by channeling effect was presented on Fig.10. Due to the fact that the $\eta$ phase contrast is similar to the contrast of $\gamma$ grains, in which planes with high atom density are perpendicular to the incident electron beam, for tomographic reconstruction the areas of grains with the lowest contrast should be selected. Otherwise, the segmentation process of selected microstructural elements is impossible or burdened with large errors. The $\eta$ phase detection capabilities in grains of varying contrast is shown in Fig.10b. Lamellar particles, identified as the $\eta$ phase by electron diffraction and EDS analysis (details in Ref. [15]), were observed at grain boundaries and occasionally at twin boundaries. It can be seen that precipitates with round-to-blocky morphology are randomly dispersed within the matrix.

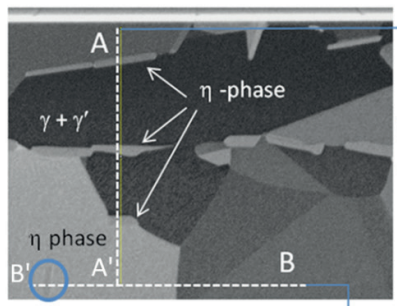

a)

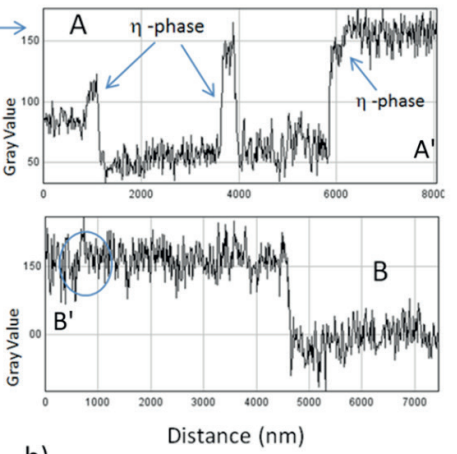

b)

Fig. 10. The influence of electron channeling effect on observed contrast of microstructural elements during SEM imaging for the 718Plus superalloy. a) Microstructure of nickel-base superalloy imaging using SEM-BSE (1,7 kV, EsB detector, EsB Ugrid = 1332 $\mathrm{V})$. The apparent differences in image contrast between the different crystallographic oriented grains caused by channeling effect. b) Linear distribution of intensity changes along selected lines marked on Fig.10a as A - A' and B - B'

Three-dimensional visualization of FIB-SEM tomographic reconstructed volume of the 718Plus superalloy microstructure containing the $\eta$ phase is seen in Fig.11. Figs $12 \mathrm{a}$ and $\mathrm{b}$ show a morphology of selected $\eta$ particles precipitated at the $\gamma$ grain boundary at different angle of view. Platelets, occasionally as a lamellar structure at grain boundaries and inside the grains, were observed. The $\eta$ phase precipitates at the $\gamma$ grain boundaries (Fig, 12a) are much thicker $(270 \mathrm{~nm})$ than the $\eta$ phase plates $(56 \mathrm{~nm})$ in lamellar precipitates inside the $\gamma$ grains (Fig. 12b). 3D visualization of tomographic reconstructed volume of the 718Plus superalloy 
at different angles of view shown in Fig. 13, reveals various shapes of carbides. In addition to the difference in morphology, these carbides also have a different chemical composition, which was visible in SEM images recorded using backscatter electrons (Fig.10). In BSE the same carbide precipitates were observed. Moreover, in 3D visualization carbides at the $\eta$ phase interfaces were identified (Fig. 12a).

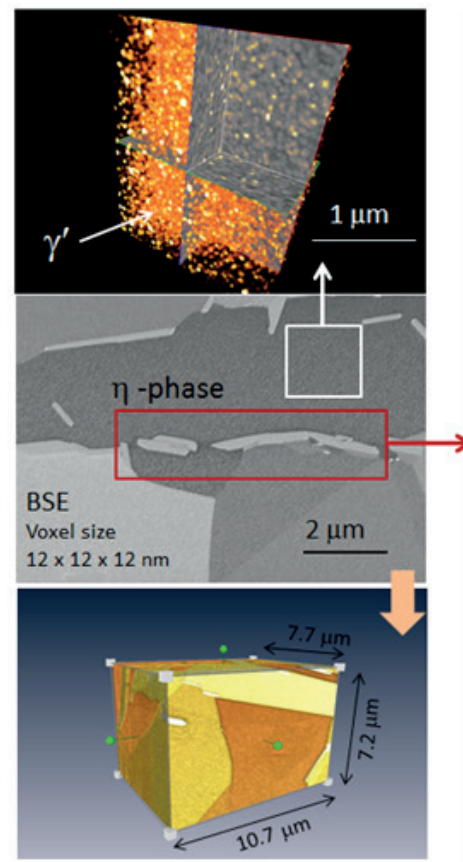

a)

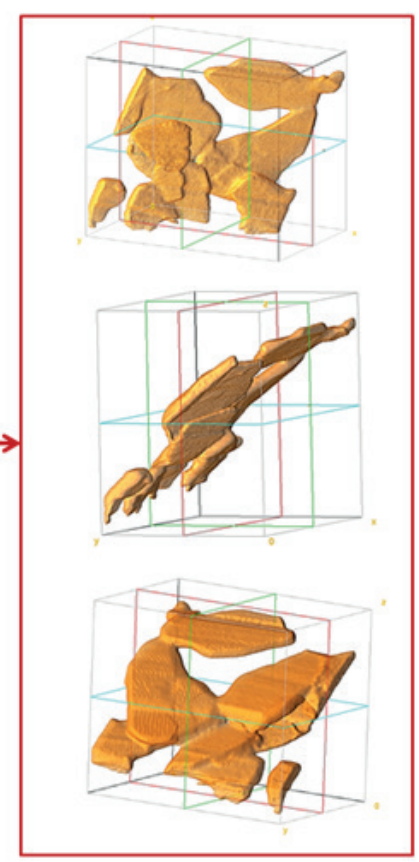

b)
Fig. 11. Microstructure of the 718Plus as seen using SEMEsB detector (middle image in Fig. 11a) and three-dimensional visualization of tomographic reconstructed volume of the 718Plus using FIB-SEM tomography technique; a) spatial distribution of spherical $\gamma^{\prime}$ precipitates observed inside the analysed volume of $\gamma$ matrix, b) morphology of $\eta$ phase particles precipitated at the $\gamma$ grains boundary at different angle of view. Reconstructed volume: $10.7 \mathrm{x}$ $7.2 \times 7.7 \mu \mathrm{m}$ and voxel size: $12 \times 12 \times 12 \mathrm{~nm}$

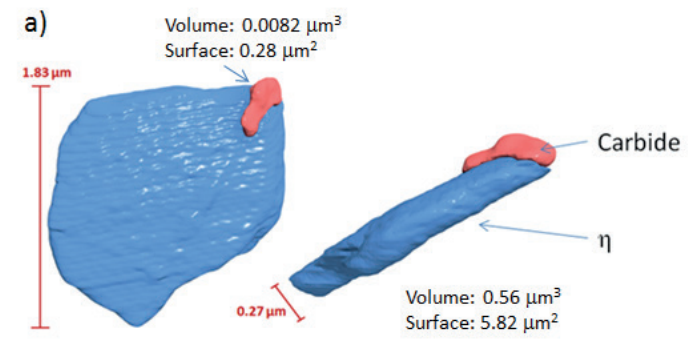

b)
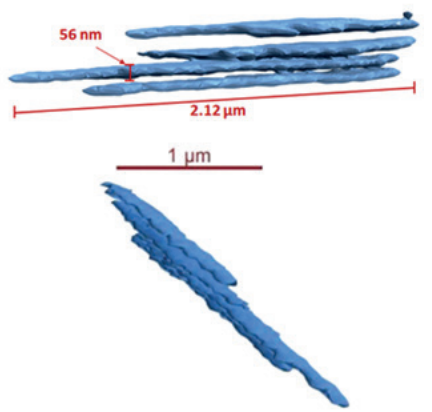

Fig. 12. 3D visualization of the $\eta$ phase in 718 Plus superalloy by FIB-SEM tomography technique; a) morphology of selected $\eta$ phase particle precipitated at the $\gamma$ grain boundary with the carbide precipitates on its interface b) morphology of lamellar $\eta$ phase particles precipitated at the $\gamma$ grain boundary at different view angle.

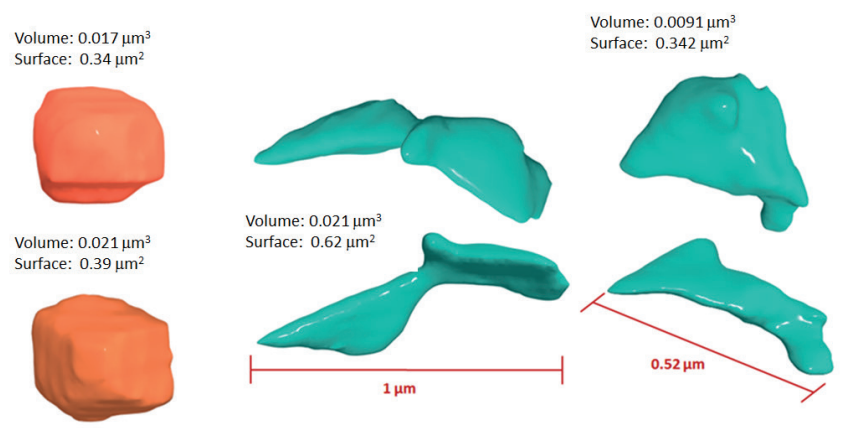

Fig. 13. 3D visualization of carbides with different shape and chemical composition observed in reconstructed volume of the 718Plus superalloy (FIB-SEM tomography). Morphology of selected carbide particles presented at different view angles

Application of tomographic techniques (STEM-EDX, FIB-SEM) allowed for visualize the precipitates in the 718Plus superalloy, their quantitative assessment, spatial distribution and morphology. The resolution in the tomographic reconstructions is limited from a few tenths of nanometres in case of TEM tomography to few nanometres in case of FIBSEM tomography. In practice, however, the resolution is still of the order of few nanometres because is limited stability of the sample holders and the presence of dynamic diffraction in crystalline solids. This is why X-ray spectroscopic imaging (STEM-EDX) which enables the mapping of local concentrations of selected chemical elements, was used for qualitative and quantitative evaluation of the strengthening particles of $\gamma^{\prime}$ phase and precipitates of the $\eta$ phase with resolution about of $0.2 \mathrm{~nm}$.

\section{Summary}

TEM investigation of the 718Plus revealed that its microstructure consists of a $\gamma$ matrix strengthened mainly by ordered face centered cubic $\gamma^{\prime}$ phase precipitates and $\eta$ phase particles of hexagonal crystal structure. Spectroscopical analyses showed that the $\gamma$ matrix contains $\mathrm{Ni}, \mathrm{Fe}, \mathrm{Co}, \mathrm{Cr}$ and $\mathrm{W}$, while $\mathrm{Ni}, \mathrm{Al}, \mathrm{Ti}$ and $\mathrm{Nb}$ partition to the $\gamma^{\prime}$ and $\eta$ phases.

Tomography investigation confirms the ability of STEMEDX and FIB-SEM tomography techniques to reconstruct 3D microstructure of alloy investigated with dimensions in tens of nanometers.

Tomographic reconstruction performed using STEM-EDX technique enabled $3 \mathrm{D}$ visualization the precipitates shapes, spatial distribution and perform qualitative and quantitative evaluation of $\gamma^{\prime}$ precipitates in the 718Plus superalloy. Due to new possibilities offered by a ChemiSTEM ${ }^{\mathrm{TM}}$ system (with a Super-X detector), high X-ray signal over a large tilt angle ( \pm $60^{\circ}$ ) of sample was achieved and series of $2 \mathrm{D}$ elemental maps were collected.

Imaging of microstructures in SEM using energy selective backscatter electron detector (EsB) allowed to obtain contrast level in grayscale between precipitates and the matrix sufficient to perform tomographic reconstruction. 
The FIB-SEM tomographic reconstruction in 3D showed that the $\eta$ phase precipitates have a different morphology: lamellar precipitates at grain boundaries and inside the grains and platelets at grain boundaries. The crystal structure of the precipitate was consistent with $\eta-\mathrm{Ni} 3 \mathrm{Ti}$ D024 phase and its chemistry indicated the $\mathrm{Ni}_{6}(\mathrm{AlTi}) \mathrm{Nb}$ phase.

In comparison to conventional electron microscopy, electron tomography techniques permit to qualify complementary information about the size of precipitates, their shape and 3D distribution in the reconstructed volume with respect to conventional particle analysis methods, e.g. quantitative TEM and SEM metallography.

Acknowledgements: The authors acknowledge the support from the AGH-UST statutory project (nr 11.11.110. 299). The authors thank B. Rutkowski, PhD, A. Gruszczyński, MSc. (AGH-UST) and A. Carlsson, PhD (FEI) for their assistance in the STEM-EDX and FIB-SEM investigations.

\section{REFERENCES}

[1] R.M. Kearsey, J. Tsang, S. Oppenheimer, E. Mcdevitt, JOM 64, 2 (2012).

[2] X. Xishan, G. Wang, J. Dong, et al., Superalloy 718, 625, 706 and Derivatives 2005, ed. by E.A. Loria, TMS (The Minerals, Metals \& Materials Society) (2005).

[3] W.D. Cao, R.L. Kennedy, Superalloys 2004, Seven Springs Conference, Seven Springs, ed. by E.A. Loria, TMS (The Minerals, Metals \& Materials Society) (2004).
[4] E.J. Pickering, H. Mathur, A. Bhowmik, et al., Acta Materialia 60, (2012).

[5] W.D. Cao, Superalloys 718, 625, 706 and Derivatives 2005, ed. by E.A. Loria, TMS (The Minerals, Metals \& Materials Society) (2005).

[6] L. Viskari, Y. Cao, M. Norell, G. Sjöberg, K. Stiller, Mater Sci Eng A 528 (2011).

[7] X. Xie, G.Wang, J. Dong, C. Xu, W. Cao, R. Kennedy, Sixth International Special Emphasis Symposium on Superalloys 718, 625, 706 and Derivatives, ed. by E.A. Loria, TMS (The Minerals, Metals \& Materials Society) (2005).

[8] Technical Data Sheet: ATI 718Plus ${ }^{\circledR}$ Alloy Precipitation Hardened Nickel-base Superalloy (UNS N07818) version 1 (2013).

[9] R.L. Kennedy, Superalloys 718, 625, 706 and Derivatives 2005, ed. by E.A. Loria TMS (The Minerals, Metals \& Materials Society) (2005).

[10] P.A. Midgley, R.E. Dunin-Borkowski, Nature Materials 8, 2 (2009).

[11] A. Kruk, A. Czyrska-Filemonowicz; Archives of Metallurgy and Materials 58, (2013)

[12] M. Weyland, P.A. Midgley, Materials Today 7, 12 (2004).

[13] M.D. Uchic, M.A. Groeber, D.M. Dimiduk, J. P. Simmons, Scr Mater. 55 (2006).

[14] P. Schlossmacher, D.O. Klenov, B. Freitag, S. von Harrach, A. Steinbach, Microscopy and Analysis 24, 7 (2010).

[15] S. Lech, MSc. thesis, AGH University of Science and Technology, Kraków (2014) 
